Preprint typeset in JHEP style - HYPER VERSION

\title{
Topological Sigma B Model in 4-Dimensions
}

\author{
Hyun-Keun Jun \\ Department of Mathematics, Kyungpook University, Daegu 702-701, Korea. \\ E-mail hyunkeun@knu.ac.kr \\ Jae-Suk Park* \\ Department of Mathematics, Yonsei University, Seoul 120-749, Korea. \\ E-Mail.jaesuk@yonsei.ac.kn
}

ABSTRACT: We propose a 4-dimensional version of topological sigma B-model, governing maps from a smooth compact 4-manifold $M$ to a Calabi-Yau target manifold $X$. The theory depends on complex structure of $X$, while is independent of Kähler metric of $X$. The theory is also a 4-dimensional topological field theory in the sense that the theory is independent of variation of Riemannian metric of the source 4-manifold $M$, potentially leading to new smooth invariant of 4-manifolds. We argue that the theory also comes with a topological family parametrized by the extended moduli space of complex structures.

Keywords: Topological Field Theory, Topological Sigma Model, Smooth Invariants of 4-Manifolds.

${ }^{*}$ This work was supported by KOSEF Interdisciplinary Research Grant No. R01-2006-000-10638-0. 


\section{Contents}

1. Introduction 1

2. Construction of Our Model 2

2.1 Global Fermionic Symmetry and Lagrangian 3

2.2 An Analogy with Topological String B Model 6

2.3 Two Important Properties

2.4 Localization and Fermionic Zero Modes 9

2.5 The Observables 10

3. Toward the Topological Family 10

\section{Introduction}

The purpose of this paper is to propose a 4-dimensional topological sigma model, which governs maps from a smooth oriented compact 4-manifold $M$ to a Calabi-Yau manifold $X$. Our model is a topological theory in 4-dimensions in the sense that the variation of action functional with respect to Riemannian metric of the source 4-manifold $M$ is BRST exact. Analogous to the topological string B-model in 2-dimensions [1] our model does not depend on variation of Kähler structures of the target Calabi-Yau space $X$, while depending on variation of complex structures of $X$. We shall also argue that there should be a family of topological theory parametrized by the extended moduli space of complex structures of $X$ similarly to the topological string B model [1, 8, 9].

Our theory may lead to potentially new smooth invariant, a family of invariants parametrized by the moduli space of complex structures of Calabi-Yau manifold, of 4manifold. Our theory has many properties similar to those of Donaldson-Witten theory [2], while a perfect analogy between the two theories seems require the target space to admit a holomorphic symplectic structure. This issue will be pursued in a forthcoming paper 沟.

This paper is organized as follows; In section 2 we briefly review some basic properties of the Atiyah class of holomorphic tangent space of Calabi-Yau space and construct the Lagrangian of our model. Then we show that the variations of our Lagrangian with respect to infinitesimal variations of both Riemannian metric of the source 4-manifold $M$ and Kähler metric of the target Calabi-Yau manifold $X$ are BRST exact. We also show that path integrals of the model are localized to the space of constant maps and construct topological observables. In section 3 we study infinitesimal deformations of the theory and argue that there should be a family of theory parametrized by the extended moduli space of complex structures of $X$. 


\section{Construction of Our Model}

Our model will be a four dimensional sigma model, which is a theory of maps from a smooth oriented Riemannian 4-manifold $M$ to a compact Calabi-Yau manifold $X$. To define our Lagrangian, we need to pick a Riemannian metric $h$ on $M$, a complex structure on $X$ as well as a Kähler metric $g$ on $X$, while the theory will depend only on the complex structure on $X$ - that is, independent to infinitesimal changes in both $h$ and $g$. This feature is similar to that of topological string B model. We begin with setting up notations and conventions.

Let $M$ be a smooth oriented compact Riemannian 4-manifold. We denote the space of differential $r$-forms on $M$ by $\Omega^{r}(M)$ and the exterior derivative on $M$ by $d$, i.e., $d$ : $\Omega^{r}(M) \rightarrow \Omega^{r+1}(M)$ satisfying $d^{2}=0$. We pick a Riemannian metric $h$ on $M$ and denote $*$ the corresponding Hodge star operator, so that $*: \Omega^{r}(M) \longrightarrow \Omega^{4-r}(M)$ and $*^{2}=1$. Then the space $\Omega^{2}(M)$ of 2 -forms is decomposed into the self-dual and anti-self-dual parts;

$$
\Omega^{2}(M)=\Omega_{+}^{2}(M) \oplus \Omega_{-}^{2}(M) .
$$

For a two form $a$ we denote $a^{+}$the projection to the self-dual part of $a$, i.e., $* a^{+}=a^{+}$.

Let $X$ be a complex $n$-dimensional compact Calabi-Yau manifold - a Käher manifold with $c_{1}(X)=0$. Let $T_{\mathbb{C}} X$ denote the complexified tangent bundle to $X$. We pick a complex structure $J, J^{2}=-1$, on $X$ and extend $J$ to a complex endomorphism of $T_{\mathbb{C}} X$ leading to the eigen-space decompositions $T_{\mathbb{C}} X=\mathcal{T} \oplus \overline{\mathcal{T}}$, where $\mathcal{T}$ is the holomorphic tangent bundle of rank $n$. Similarly the complexified cotangent bundle $T_{\mathbb{C}}^{*} X$ has the decomposition $T_{\mathbb{C}}^{*} X=\mathcal{T}^{*} \oplus \overline{\mathcal{T}}^{*}$, where $\mathcal{T}^{*}$ is the holomorphic cotangent bundle. We denote the space of differential $(p, q)$-forms on $X$ by $\Omega^{p, q}(X)=\Omega^{0, q}\left(X, \wedge^{p} \mathcal{T}^{*}\right)$. The exterior derivative $d$ is decomposed as $d=\partial+\bar{\partial}$, where $\bar{\partial}: \Omega^{p, q}(X) \longrightarrow \Omega^{p+1, q}(X)$ and $\bar{\partial}^{2}=0$. We also consider the space $\Omega^{0, q}\left(X, \wedge^{p} \mathcal{T}\right)$ of $(0, q)$-form on $X$ valued in the section of $\wedge^{p} \mathcal{T}$. Let $\Omega$ be a choice of no-where vanishing holomorphic $n$-form on $X$. Then we have the familiar isomorphism

$$
\Omega^{0, q}\left(X, \wedge^{p} \mathcal{T}\right) \simeq \Omega^{n-p, q}(X)=\Omega^{0, q}\left(X, \wedge^{n-p} \mathcal{T}^{*}\right)
$$

Pick a Kähler metric $g$ and let $\widetilde{\nabla}$ be the unique $g$-connection of $\mathcal{T}$ such that the $(0,1)$ part of connection is $\bar{\partial}$. Let $\nabla$ be the canonical $(1,0)$-part of the metric connection on $\mathcal{T}$ such that the $(2,0)$-part of the curvature 2 -form vanishes;

$$
[\nabla, \nabla]=0
$$

Then the curvature $R$ of the total connection $\widetilde{\nabla}=\nabla+\bar{\partial}$ is an $(1,1)$-form on $X$ valued in $\operatorname{End}(\mathcal{T})$

$$
R=[\bar{\partial}, \nabla] \in \Omega^{1,1}(\operatorname{End}(\mathcal{T})) .
$$

Pick a local holomorphic coordinates $\left\{u^{i}\right\}, i=1,2, \ldots n$, and their complex conjugates $\left\{u^{\bar{i}}\right\}$. Denoting the Kähler metric $g$ in terms of local coordinates by $g_{i \bar{j}}$, the associated connection $(1,0)$-form is

$$
\Gamma^{i}{ }_{j k} d z^{k}:=g^{i \bar{\ell}} \partial_{j} g_{k \bar{\ell}} d u^{k}
$$


where $\partial_{j}=\frac{\partial}{\partial u^{j}}$. Note that $\Gamma^{i}{ }_{j k}=\Gamma^{i}{ }_{k j}$. Then the curvature $R$ of $\mathcal{T}$ in components is given by

$$
R^{i}{ }_{j k \bar{\ell}}=\partial_{\bar{\ell}} \Gamma^{i}{ }_{j k} .
$$

It follows that $R^{i}{ }_{j k \bar{\ell}}=R^{i}{ }_{k j \bar{\ell}}$, so that $R \in \Omega^{0,1}\left(X, \operatorname{Hom}\left(S^{2} \mathcal{T}, \mathcal{T}\right)\right)$. It is also obvious that $\bar{\partial} R=0$. On the other hand, $\nabla R \neq 0$ in general, leading to the "higher curvature" tensor $S \in \Omega^{0,1}\left(X, H \operatorname{Hom}\left(S^{2} \mathcal{T} \otimes \mathcal{T}, \mathcal{T}\right)\right.$ defined by $S:=[\nabla, R]$

$$
S_{m j k \bar{\ell}}^{i}:=\nabla_{m} R^{i}{ }_{j k \bar{\ell}} .
$$

From the relation (2.3), it follows that $S^{i}{ }_{m j k \bar{\ell}}$ is actually symmetric in all three indices $m, j, k$. We recall that $\bar{\partial} R=0$ due to the definition (2.4), which condition in components is

$$
\partial_{\bar{m}} R_{j k \bar{\ell}}^{i} \psi^{\bar{m}} \psi^{\bar{\ell}}=0
$$

where we used anti-commuting variables $\psi^{\bar{i}}$, for a convenience, and the Einstein summation conventions (to be used throughout this paper). On the other hand $S$ is not $\bar{\partial}$-closed in general. We have

$$
\partial_{\bar{n}}\left(S_{m j k \bar{\ell}}^{i}\right) t^{j} t^{k} \psi^{\bar{n}} \psi^{\bar{\ell}}=\left(R_{p m \bar{n}}^{i} R^{p}{ }_{j k \bar{\ell}}+2 R_{p j \bar{n}}^{i} R_{\bar{k} m \bar{\ell}}^{p}\right) t^{j} t^{k} \psi^{\bar{n}} \psi^{\bar{\ell}},
$$

where we further used commuting variables $t^{i}$, for a convenience. We remark that the identity (2.9) was derived in the appendix of the paper [5]. We also remark that the curvature $R$ and the higher curvature $S$ are the Dolbeault representatives of the Atiyah class and a higher Atiyah class of $\mathcal{T}$, which are the lowest parts of the sequence of higher

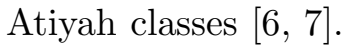

\subsection{Global Fermionic Symmetry and Lagrangian}

Our model is a theory of maps $\phi: M \rightarrow X$ from the Riemanian 4-manifold $M$ to the Calabi-Yau manifold $X$, as are specified in the above, with the choices of a Riemannian metric on $M$, a complex structure on $X$ and a Käher metric on $X$. A map $\phi: M \rightarrow X$ can be described locally by functions $u^{i}(x)$ and their complex conjugates $u^{\bar{i}}(x)$, where $x$ denotes a a point in $M$ and the $u^{i}$ corresponds to holomorphic coordinates on $X$. We shall denote the corresponding bosonic fields by $u^{i}$ and $u^{\bar{i}}$, which are 0-forms on $M$. We introduce an integral valued quantum number called ghost number $U$ and assign $U=0$ to the fields $\left(u^{i}, u^{\bar{i}}\right)$. We introduce an anti-commuting 0 -form field $\psi^{\bar{i}}$ of $U=1$, which is a section of $\phi^{*}(\overline{\mathcal{T}})$, the pullback of anti-holomorphic tangent bundle of $X$ to $M$. We also introduce another anti-commuting 0 -form field $\eta_{i}$ with $U=3$, a section of $\phi^{*}\left(\mathcal{T}^{*}\right)$. We further introduce an anti-commuting 1-form field $\rho^{i}=\rho_{\alpha}^{i} d x^{\alpha}$ of $U=-1$ and a commuting self-dual 2-form field $H^{i}=\frac{1}{2} H_{\alpha \beta}^{i} d x^{\alpha} \wedge d x^{\beta}$ of $U=-2$ - the both fields are sections of $\phi^{*}(\mathcal{T})$. In the above and throughout this paper $\alpha, \beta=1,2,3,4$ are tangent indices to $M$ and $\left\{x^{\alpha}\right\}$ denotes coordinates on $M$. We also introduce a commuting 1-form field $A_{i}=A_{i \alpha} d x^{\alpha}$ of $U=2$ and an anti-commuting self-dual 2-form field $\chi_{i}=\frac{1}{2} \chi_{i \alpha \beta} d x^{\alpha} \wedge d x^{\beta}$ of $U=1$-the both fields are sections of $\phi^{*}\left(\mathcal{T}^{*}\right)$. Finally we require two more 0 -form fields $\left(\bar{\eta}^{i}, v^{i}\right)$ of $U=(-3,-2)$, which are sections of $\phi^{*}\left(\mathcal{T}^{i}\right)$. A field with even ghost number $U$ is commuting and a field with odd ghost number $U$ is anti-commuting. We summarize all the fields in the following table; 


\begin{tabular}{|c|c|c|c|c|c|c|c|}
\hline form degree $\backslash$ ghost number & -3 & -2 & -1 & 0 & 1 & 2 & 3 \\
\hline$\Omega^{0}(M)$ & $\bar{\eta}^{i}$ & $v^{i}$ & & $u^{i}, u^{i}$ & $\psi^{i}$ & & $\eta_{i}$ \\
\hline$\Omega^{1}(M)$ & & & $\rho^{i}$ & & & $w_{i}$ & \\
\hline$\Omega_{+}^{2}(M)$ & & $H^{i}$ & & & $\chi_{i}$ & & \\
\hline
\end{tabular}

Table 1: The form degree on $M$ and the ghost numbers of the various fields

For a later purpose we state that the indices tangent to $M$ are raised and lowered by a Riemannian metric $h_{\alpha \beta}$ on $M$. Then the self-duality conditions of the two 2-form fields $\chi_{i}=\frac{1}{2} \chi_{i \alpha \beta} d x^{\alpha} \wedge d x^{\beta}$ and $H^{i}=\frac{1}{2} H_{\alpha \beta}^{i} d x^{\alpha} \wedge d x^{\beta}$ are

$$
\begin{aligned}
H_{\alpha \beta}^{i} & =\frac{1}{2} \varepsilon_{\alpha \beta \gamma \delta} h^{\alpha \gamma^{\prime}} h^{\delta \delta^{\prime}} H_{\gamma^{\prime} \delta^{\prime}}^{i}, \\
\chi_{i \alpha \beta} & =\frac{1}{2} \varepsilon_{\alpha \beta \gamma \delta} h^{\gamma \gamma^{\prime}} h^{\delta \delta^{\prime}} \chi_{i \gamma^{\prime} \delta^{\prime}} .
\end{aligned}
$$

Now we state conventions on covariant derivatives. A connection on $\phi^{*}(\overline{\mathcal{T}})$ is obtained by pulling back the metric connection of $\overline{\mathcal{T}}$ from $X$ to $M$, giving the covariant derivative of $\psi^{\bar{i}}$

$$
\bar{D} \psi^{\bar{i}}=d \psi^{\bar{i}}+d u^{\bar{j}} \Gamma_{\bar{j} \bar{k}}^{\bar{i}} \psi^{\bar{k}},
$$

where $\Gamma^{\bar{i}}{ }_{\bar{j} \bar{k}}=g^{\bar{i} \ell} \partial_{\bar{j}} g_{\ell \bar{k}}$, and similarly for any other section of $\phi^{*}(\overline{\mathcal{T}})$. A connection on $\phi^{*}\left(\mathcal{T}^{*}\right)$ is obtained by pulling back the metric connection of $\mathcal{T}^{*}$ from $X$ to $M$, giving the covariant derivative of $\eta_{i}$

$$
D \eta_{i}=d \eta_{i}-d u^{j} \Gamma^{k}{ }_{i j} \eta_{k}
$$

and similarly for any other section of $\phi^{*}\left(\mathcal{T}^{*}\right)$. We also introduce a twisted covariant derivative $\mathfrak{D}$ acting on the various sections of $\phi^{*}\left(\mathcal{T}^{*}\right)$ by the formula

$$
\begin{aligned}
\mathfrak{D} \eta_{i} & =D \eta_{i}-R_{i j \bar{\ell}}^{k} \psi^{\bar{\ell}} \rho^{j} \eta_{k}, \\
\mathfrak{D} w_{i} & =D w_{i}-R_{i j \bar{\ell}}^{k} \psi^{\bar{\ell}} \rho^{j} \wedge w_{k} .
\end{aligned}
$$

As the rationale behind the above definition, we first recall that the field $\rho^{i}$ is an anticommuting 1-form on $M$. The twisted covariant derivative $\mathfrak{D}$ can be compared with the usual gauge covariant derivative in Yang-Mills theory on $M$, where the exterior derivative is replaced with $D$, the gauge 1 -form connection is replaced with $\rho^{i}$ and the structure constant is replaced with $R^{k}{ }_{i j \bar{\ell}} \psi^{\bar{\ell}}$, and the Bianchi identity is replaced with the identity in (2.9). We should remark that the closely related thing happens in the Rozanky-Witten-KontsevichKapranov theory, which is a 3 -dimensional topological sigma model with a holomorphic symplectic manifold as the target space.

Our model has a global fermionic symmetry. Before stating its transformation laws, it is convenient to divide all the fields into four kinds of multiplets;

- $\left(u^{i}, \rho^{i}, H^{i}\right)$ with $U=(0,-1,-2)$ in $\left(\Omega^{0}(M), \Omega^{1}(M), \Omega_{+}^{2}(M)\right)$.

- $\left(u^{\bar{i}}, \psi^{\bar{i}}\right)$ with $U=(0,1)$ both in $\Omega^{0}(M)$. 
- $\left(\eta_{i}, w_{i}, \chi_{i}\right)$ with $U=(3,2,1)$ in $\left(\Omega^{0}(M), \Omega^{1}(M), \Omega_{+}^{2}(M)\right)$.

- $\left(\bar{\eta}^{i}, v^{i}\right)$ with $U=(-3,-2)$ both in $\Omega^{0}(M)$.

We first postulate the fermionic transformation laws for $\left(u^{i}, \rho^{i}, H^{i}\right)$ and $\left(u^{\bar{i}}, \psi^{\bar{i}}\right)$

$$
\begin{aligned}
Q u^{i} & =0, \\
Q \rho^{i} & =d u^{i}, \\
Q H^{i} & =-\left(D \rho^{i}+\frac{1}{2} R_{j k \bar{\ell}}^{i} \rho^{j} \wedge \rho^{k} \psi^{\bar{\ell}}\right)^{+}, \\
Q u^{\bar{i}} & =\psi^{\bar{i}}, \\
Q \psi^{\bar{i}} & =0 .
\end{aligned}
$$

We also postulate that

$$
\begin{aligned}
Q \eta_{i}= & 0, \\
Q w_{i}= & -\mathfrak{D} \eta_{i}, \\
Q \chi_{i}= & \left(\mathfrak{D} w_{i}\right)^{+}-R^{k}{ }_{i j \bar{\ell}} H^{j} \psi^{\bar{\ell}} \eta_{k} \\
& -R^{k}{ }_{i j \bar{\ell}}\left(d u^{\bar{\ell}} \wedge \rho^{j}\right)^{+} \eta_{k} \\
& +\frac{1}{2} S^{n}{ }_{i j k \bar{\ell}}\left(\rho^{j} \wedge \rho^{k}\right)^{+} \psi^{\bar{\ell}} \eta_{n}, \\
Q \bar{\eta}^{i}= & v^{i} \\
Q v^{i}= & 0
\end{aligned}
$$

for $\left(\eta_{i}, w_{i}, \chi_{i}\right)$ and $\left(\bar{\eta}^{i}, v^{i}\right)$. The global fermionic supercharge $Q$ carries $U=1$ and satisfies $Q^{2}=0$. The property $Q^{2}=0$ may be checked explicitly - the only non-trivial cases are for $Q^{2} H^{i}$ and $Q^{2} \chi_{i}$ and a little tedious but straightforward computations show that

$$
\begin{aligned}
Q^{2} H^{i}= & \frac{1}{2} \partial_{\bar{n}} R_{j k \bar{\ell}}^{i} \psi^{\bar{n}} \psi^{\bar{\ell}} \rho^{j} \wedge \rho^{k} \\
Q^{2} \chi_{i}= & \left(\partial_{\ell} \Gamma_{i j}^{k}-\Gamma_{\ell i}^{p} \Gamma_{p j}^{k}\right) d u^{\ell} \wedge d u^{j} \eta_{k} \\
& +\left(\partial_{m} R_{i j \bar{\ell}}^{k}-\Gamma_{m j}^{p} R_{i p \bar{\ell}}^{k}-\Gamma_{m i}^{p} R_{p j \bar{\ell}}^{k}+\Gamma_{m p}^{k} R_{i j \bar{\ell}}^{p}-S_{m j i \bar{\ell}}^{k}\right) d u^{m} \psi^{\bar{\ell}} \rho^{j} \eta_{k} \\
& +\frac{1}{2}\left(\partial_{\bar{n}} S_{i j k \bar{\ell}}^{m}-R_{p i \bar{n}}^{m} R_{j k \bar{\ell}}^{p}-R_{p j \bar{n}}^{m} R_{k i \bar{\ell}}^{p}-R_{p k \bar{n}}^{m} R_{i j \bar{\ell}}^{p}\right) \psi^{\bar{n}} \psi^{\bar{\ell}} \rho^{j} \wedge \rho^{k} \eta_{m}
\end{aligned}
$$

The condition $Q^{2} H^{i}=0$ follows from the condition $\bar{\partial} R=0$, eq. (2.8), (note that $\psi^{\bar{n}} \psi^{\bar{\ell}}=$ $\left.-\psi^{\bar{\ell}} \psi^{\bar{n}}\right)$. To show $Q^{2} \chi_{i}=0$, we use the condition $[\nabla, \nabla]=0$, eq. (2.3), (note that $d u^{\ell} \wedge d u^{j}=-d u^{j} \wedge d u^{\ell}$ ), and the definition $S_{m j k \bar{\ell}}^{i}=\nabla_{m} R_{j k \bar{\ell}}^{i}$, eq. (2.7), as well as the "Bianchi identity", eq. (2.9) (note that $\rho^{j} \wedge \rho^{k}=\rho^{k} \wedge \rho^{j}$ since $\rho^{i}$ is an anti-commuting 1-form).

Now it is not difficult to construct our Lagrangian $\mathscr{L}$ of $U=0$, which is invariant under $Q$ and is covariant on $M$. We take

$$
\mathscr{L}=Q V
$$


where

$$
V=\int_{M}\left(\chi_{i} \wedge H^{i}+g_{i \bar{j}} \rho^{i} \wedge * d u^{\bar{j}}+\bar{\eta}^{i} * \mathfrak{D}^{*} w_{i}\right) .
$$

We note that $V$ is covariant on $M$ and of $U=-1$. It follows that $\mathscr{L}=Q V$ is $Q$-invariant and of $U=0$. The explicit form of the Lagrangian $\mathscr{L}$ is given by

$$
\begin{aligned}
\mathscr{L}=\int_{M} & \left(g_{i \bar{j}} d u^{i} \wedge * d u^{\bar{j}}-\rho^{i} \wedge * \bar{D} \psi^{\bar{j}} g_{i \bar{j}}+\chi_{i} \wedge\left(D \rho^{i}+\frac{1}{2} R^{i}{ }_{j k \bar{\ell}} \rho^{j} \wedge \rho^{k} \psi^{\bar{\ell}}\right)^{+}\right. \\
& +H^{i} \wedge \mathfrak{D}^{+} w_{i}+R^{k}{ }_{i j \bar{\ell}} \eta_{k} H^{i} \wedge H^{j} \psi^{\bar{\ell}}-R^{k}{ }_{i j \bar{\ell}} \eta_{k} H^{i} \wedge\left(\rho^{j} \wedge d u^{\bar{\ell}}\right)^{+} \\
& \left.-\frac{1}{2} S_{i j k \bar{\ell}}^{n} \eta_{n} H^{i} \wedge\left(\rho^{j} \wedge \rho^{k}\right)^{+} \psi^{\bar{\ell}}+v^{i} * \mathfrak{D}^{*} w_{i}+\bar{\eta}^{i} * \mathfrak{D}^{*} \mathfrak{D} \eta_{i}\right) .
\end{aligned}
$$

\subsection{An Analogy with Topological String B Model}

Now we want to discuss an analogy with the Topological String B model [1]. Let $\Sigma$ be a smooth oriented compact Riemannian surface. We denote the space of differential $r$-form on $\Sigma$ by $\Omega^{r}(\Sigma)$ and the the exterior derivative on $\Sigma$ also by $d$. We pick a Riemannian metric $h$ on $\Sigma$ and denote $*$ the Hodge star operator on $\Sigma$. We first note that the space 2 -form on $\Sigma$ is isomorphic the space of 0 -form.

The B model governs maps $\phi: \Sigma \rightarrow X$ with the choice of a Riemannian metric $h$ on $\Sigma$, a Käher metric $g_{i \bar{j}}$ on $X$ and a complex structure $J$ on $X$. We adopt the same conventions involving the target Calabi-Yau space $X$ as in our model. Picking a local holomorphic coordinates $\left\{u^{i}\right\}$, a map $\phi$ can be described locally via local coordinates $\left(u^{i}, u^{\bar{i}}\right)$ on $X$ regarded as functions on $\Sigma$. We have an integral valued quantum number called ghost number $U$ and assign $U=0$ to the bosonic fields $\left(u^{i}, u^{\bar{i}}\right)$. We have an anti-commuting 0 -form field $\psi^{\bar{i}}$ of $U=1$, a section of $\phi^{*}(\overline{\mathcal{T}})$, the pullback of anti-holomorphic tangent bundle of $X$ to $M$. We also have an anti-commuting 1-form field $\rho^{i}$ of $U=-1$, a section of $\phi^{*}(\mathcal{T})$. Finally we have a anti-commuting 0 -form field $\chi_{i}$ of $U=1$, a section of $\phi^{*}\left(\mathcal{T}^{*}\right)$. The fermionic transformation laws are

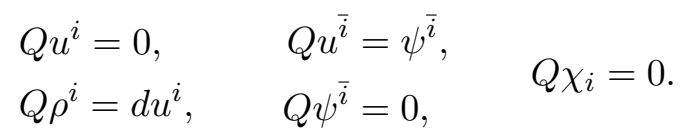

The global fermionic supercharge $Q$ carries $U=1$ and satisfies $Q^{2}=0$. The Lagrangian is given by

$$
\mathscr{L}_{B}=\int_{\Sigma}\left(g_{i \bar{j}} d u^{i} \wedge * d u^{\bar{j}}-\rho^{i} \wedge * \bar{D} \psi^{\bar{j}} g_{i \bar{j}}+\chi_{i}\left(D \rho^{i}+\frac{1}{2} R_{j k \bar{\ell}}^{i} \rho^{j} \wedge \rho^{k} \psi^{\bar{\ell}}\right)\right) .
$$

We note that the 0 -form field $\chi_{i}$ on $\Sigma$ can be dualized to a 2 -form field on $\Sigma$. Now a straightforward generalization to 4-dimensional sigma model might be obtained by declaring $\chi_{i}$ to be a self-dual 2-form field on 4-manifold $M$ while maintaining the form degrees and ghost numbers of the all the other fields;

$$
\mathscr{L}^{\prime}=\int_{M}\left(g_{i \bar{j}} d u^{i} \wedge * d u^{\bar{j}}-\rho^{i} \wedge * \bar{D} \psi^{\bar{j}} g_{i \bar{j}}+\chi_{i} \wedge\left(D \rho^{i}+\frac{1}{2} R_{j k \bar{\ell}}^{i} \rho^{j} \wedge \rho^{k} \psi^{\bar{\ell}}\right)^{+}\right) .
$$


An obvious problem of the above Lagrangian is that the field $\chi_{i}$, now a self-dual 2 -form on $M$ has a new gauge degrees of freedom. Our Lagrangian $\mathscr{L}$ in (2.18) may viewed as a result of removing such degeneracy as follows. The additional 1-form field $w_{i}$ with $U=2$ is interpreted as the ghost for the new gauge degree of freedom of the self-dual 2-form field $\chi_{i}$ of $U=1$, while the self-dual 2-form field $H^{i}$ with $U=-2$ is an auxiliary field for its gauge fixing - as is shown in the term $H^{i} \wedge \mathfrak{D}^{+} w_{i}$ in our Lagrangian $\mathscr{L}$. Being an 1-form field $w_{i}$ has residual gauge degree of freedom and the additional 0 -form field $\eta_{i}$ with $U=3$ is the ghost for the residual gauge degree of freedom, while the additional 0 -form field $\bar{\eta}^{i}$ with $U=-3$ is the antighost of the ghost $\eta^{i}$ and the additional 1-form field $v^{i}$ with $U=-2$ is the auxiliary field for its gauge fixing - as is shown in the terms $v^{i} * \mathfrak{D}^{*} w_{i}+\bar{\eta}^{i} * \mathfrak{D} * \mathfrak{D} \eta_{i}$ in our Lagrangian $\mathscr{L}$.

Besides from the above formal comparison our model will be shown to have those key properties of the topological string B model that the theory is independent of both the Riemannian metric $h$ on $\Sigma$ and the Kähler metric $g_{i \bar{j}}$ on $X$ but depends on the complex structure on the target space $X$.

\subsection{Two Important Properties}

Our Lagrangian $\mathscr{L}$ in (2.18) depends on the Riemannian metric $h_{\alpha \beta}$ on $M$ via the associated Hodge star $*$ operator, the Kähler metric $g_{i \bar{j}}$ on $X$, as well as the complex structure $J$ on $X$. In this subsection we will show that the infinitesimal variations of $\mathscr{L}$ with respect to both the Riemannian metric $h_{\alpha \beta}$ on $M$ and the Kähler metric $g_{i \bar{j}}$ on $X$ are $Q$-exact so that the partition function of the theory and the expectation values of $Q$-invariant observables depend only on the complex structure on $X$. In particular the partition function and the expectation values of $Q$-invariant observables are, following Witten's arguments in Donaldson-Witten theory [2], smooth invariants of 4-manifold.

We first establish that the (4-dimensional) energy-momentum tensor $T_{\alpha \beta}$ is $Q$-exact. $T_{\alpha \beta}$ is defined in terms of the variation of the Lagrangian with respect to an infinitesimal change of the 4-dimensional Riemannian metric $h^{\alpha \beta} \longrightarrow h^{\alpha \beta}+\delta h^{\alpha \beta}$ such that

$$
\delta \mathscr{L}=\frac{1}{2} \int_{M} \sqrt{h} \delta h^{\alpha \beta} T_{\alpha \beta},
$$

where $h$ denotes the determinant of $h_{\alpha \beta}$. The computation is straightforward besides from

a slight subtlety that the two 2-form fields $\chi_{i}=\frac{1}{2} \chi_{i \alpha \beta} d x^{\alpha} \wedge d x^{\beta}$ and $H^{i}=\frac{1}{2} H_{\alpha \beta}^{i} d x^{\alpha} \wedge d x^{\beta}$ among the all fields are subjected to the self-duality conditions (2.10). Thus the selfduality of those fields should be preserved when computing the variation $(2.21)$, as in the similar situation of Donaldson-Witten theory. So an infinitesimal change $\delta h^{\alpha \beta}$ must be accompanied by the variations

$$
\begin{aligned}
& \delta H_{\alpha \beta}^{i}=\frac{1}{2} \varepsilon_{\alpha \beta \gamma \delta} \delta h^{\gamma \gamma^{\prime}} h^{\delta \delta^{\prime}} H_{\gamma^{\prime} \delta^{\prime}}^{i}-\frac{1}{8}\left(\delta h^{\sigma \tau} h_{\sigma \tau}\right) \varepsilon_{\alpha \beta \gamma \delta} \delta h^{\gamma \gamma^{\prime}} h^{\delta \delta^{\prime}} H_{\gamma^{\prime} \delta^{\prime}}^{i}, \\
& \delta \chi_{i \alpha \beta}=\frac{1}{2} \varepsilon_{\alpha \beta \gamma \delta} \delta h^{\gamma \gamma^{\prime}} h^{\delta \delta^{\prime}} \chi_{i \gamma^{\prime} \delta^{\prime}}-\frac{1}{8}\left(\delta h^{\sigma \tau} h_{\sigma \tau}\right) \varepsilon_{\alpha \beta \gamma \delta} \delta h^{\gamma \gamma^{\prime}} h^{\delta \delta^{\prime}} \chi_{i \gamma^{\prime} \delta^{\prime}} .
\end{aligned}
$$


Then a tedious but straightforward computation gives

$$
\begin{aligned}
T_{\alpha \beta}= & 2\left(\partial_{\alpha} u^{i} \partial_{\beta} u^{\bar{j}} g_{i j}-\rho_{\alpha}^{i} \bar{D}_{\beta} \psi^{\bar{j}} g_{i \bar{j}}+\lambda^{i} \mathfrak{D}_{\alpha} w_{i \beta}+\bar{\eta}^{i} \mathfrak{D}_{\alpha} \mathfrak{D}_{\beta} \eta_{i}\right) \\
& -h_{\alpha \beta}\left(g_{i \bar{j}} \partial_{\gamma} u^{i} \partial^{\gamma} u^{\bar{j}}-\rho_{\gamma}^{i} \bar{D}^{\gamma} \psi^{\bar{j}} g_{i \bar{j}}+\lambda^{i} \mathfrak{D}_{\gamma} A_{i}^{\gamma}+\bar{\eta}^{i} \mathfrak{D}_{\gamma} \mathfrak{D}^{\gamma} \eta_{i}\right) \\
& +2 \chi_{i \alpha \gamma}\left(D_{\beta} \rho^{i \gamma}-D^{\gamma} \rho_{\beta}^{i}+R_{j k \bar{\ell}}^{i} \rho_{\alpha}^{j} \rho_{\beta}^{k} \psi^{\bar{\ell}}\right) \\
& -h_{\alpha \beta} \chi_{i \gamma \delta}\left(D^{\gamma} \rho^{i \delta}-D^{\delta} \rho^{i \gamma}+R_{j k \bar{\ell}}^{i} \rho^{j \gamma} \rho^{k \delta} \psi^{\bar{\ell}}\right) \\
& +2\left(\mathfrak{D}_{\alpha} \varphi_{i \gamma}-\mathfrak{D}_{\gamma} \varphi_{i \alpha}+R_{i j \bar{\ell}}^{k} \eta_{k} H_{\alpha \gamma}^{j} \psi^{\bar{\ell}}-S^{n}{ }_{i j k \bar{\ell}} \eta_{n} \rho_{\alpha}^{j} \rho_{\gamma}^{k} \psi^{\bar{\ell}}\right. \\
& \left.\quad+R^{k}{ }_{i j \bar{\ell}} \eta_{k}\left(\partial_{\alpha} u^{\bar{\ell}} \rho_{\gamma}^{j}-\partial_{\gamma} u^{\bar{\ell}} \rho_{\alpha}^{j}\right)\right) H_{\beta}^{i \gamma} \\
& -h_{\alpha \beta}\left(\mathfrak{D}_{\gamma} w_{i \delta}-\mathfrak{D}_{\delta} w_{i \gamma}+R^{k}{ }_{i j \bar{\ell}} H_{\gamma \delta}^{j} \psi^{\bar{\ell}}-S^{n}{ }_{i j k \bar{\ell}} \eta_{n} \rho_{\gamma}^{j} \rho_{\delta}^{k} \psi^{\bar{\ell}}\right. \\
& \left.+R^{k}{ }_{i j \bar{\ell}} \eta_{k}\left(\partial_{\gamma} u^{\bar{\ell}} \rho_{\delta}^{j}-\partial_{\delta} u^{\bar{\ell}} \rho_{\gamma}^{j}\right)\right) H^{i \delta \gamma}
\end{aligned}
$$

We note that the variations (2.22) commutes with $Q$ since $Q$ transforms as a scalar in 4-dimensions, so that it does not depend on the Riemannian metric and the application of $Q$ to a self-dual 2-form gives a self-dual 2-form on $M$. That is

$$
\begin{aligned}
Q \delta H_{\alpha \beta}^{i} & =\frac{1}{2} \varepsilon_{\alpha \beta \gamma \delta} \delta h^{\gamma \gamma^{\prime}} h^{\delta \delta^{\prime}} Q H_{\gamma^{\prime} \delta^{\prime}}^{i}-\frac{1}{8}\left(\delta h^{\sigma \tau} h_{\sigma \tau}\right) \varepsilon_{\alpha \beta \gamma \delta} \delta h^{\gamma \gamma^{\prime}} h^{\delta \delta^{\prime}} Q H_{\gamma^{\prime} \delta^{\prime}}^{i} \\
& =\delta Q H_{\alpha \beta}^{i},
\end{aligned}
$$

and $Q \delta \chi_{i \alpha \beta}=\delta Q \chi_{i \alpha \beta}$ by the same reason. All the other fields, being either 0-form or 1form fields, do not depend on metric on $M$. Thus we have $[Q, \delta]($ any fields $)=0$. Recalling that our Lagrangian is given by $\mathscr{L}=Q V$, we define $\lambda_{\alpha \beta}$ in terms of the variation of $V$ (2.2) with respect to $h^{\alpha \beta} \longrightarrow h^{\alpha \beta}+\delta h^{\alpha \beta}$ such that

$$
\delta V=\frac{1}{2} \int_{M} \sqrt{h} \delta h^{\alpha \beta} \lambda_{\alpha \beta} .
$$

From $\delta \mathscr{L} \equiv \delta Q V=Q \delta V$ we conclude that

$$
T_{\alpha \beta}=Q \lambda_{\alpha \beta}
$$

A direct computation $\delta V$ with respect to $h^{\alpha \beta} \rightarrow h^{\alpha \beta}+\delta h^{\alpha \beta}$ accompanying the variations $(2.22)$ gives us

$$
\begin{aligned}
\lambda_{\alpha \beta}= & \chi_{i \alpha \gamma} H_{\beta}^{i \gamma}+\chi_{i \beta \gamma} H_{\alpha}^{i \gamma}-\frac{1}{2} h_{\alpha \beta} \chi_{i \gamma \delta} H^{i \gamma \delta} \\
& +\bar{\eta}^{i} \mathfrak{D}_{\alpha} w_{i \beta}+\bar{\eta}^{i} \mathfrak{D}_{\beta} w_{i \alpha}-\bar{\eta}^{i} \mathfrak{D}_{\gamma} w_{i}^{\gamma} \\
& +\rho_{\alpha}^{i} \cdot \partial_{\beta} u^{\bar{j}} g_{i \bar{j}}+\rho_{\beta}^{i} \cdot \partial_{\alpha} u^{\bar{j}} g_{i \bar{j}}-h_{\alpha \beta} \rho_{\gamma}^{i} \cdot \partial^{\gamma} u^{\bar{j}} g_{i \bar{j}}
\end{aligned}
$$

One can also check explicitly that $T_{\alpha \beta}=Q \lambda_{\alpha \beta}$.

Now we want to establish that our theory is independent of the Kähler metric of the target space $X$. For this, we study the variation $\delta \mathscr{L}$ of the Lagrangian $\mathscr{L}$ with respect 
to an infinitesimal change of the Kähler metric $g^{i \bar{j}} \rightarrow g^{i \bar{j}}+\delta g^{i \bar{j}}$ preserving the Kählerian condition. Such a variation is accompanied with the following induced infinitesimal variations

$$
\begin{aligned}
\delta \Gamma^{i}{ }_{j k} & =g^{\bar{n} i} \partial_{j} \delta g_{\bar{n} k}+\delta g^{\bar{n} i} \partial_{j} g_{\bar{n} k}, \\
\delta R^{i}{ }_{j k \bar{\ell}} & =\partial_{\bar{\ell}} \delta \Gamma^{i}{ }_{j k}, \\
\delta S_{j k \ell \bar{m}}^{i} & =\nabla_{j}\left(\partial_{\bar{m}} \delta \Gamma^{i}{ }_{k \ell}\right)-\delta \Gamma_{j n}^{i} R_{k \ell \bar{m}}^{n}-\delta \Gamma^{n}{ }_{j k} R_{n \ell \bar{m}}^{i} .
\end{aligned}
$$

It may not be obvious if such variation $\delta \mathscr{L}$ is $Q$-exact since the fermionic transformation laws for the fields $H^{i}, w_{i}$ and $\chi_{i}$ depend on the Kähler metric $g_{i \bar{j}}$ on $X$. But a direct computation shows that

$$
\begin{aligned}
\delta \mathscr{L}=Q \int_{M}( & -\frac{1}{2} \delta \Gamma_{j k}^{i} \chi_{i} \wedge \rho^{j} \wedge \rho^{k}+\delta \Gamma_{i j}^{k} H^{i} \wedge A_{k} \wedge \rho^{j}-\delta \Gamma_{i j}^{k} \eta_{k} H^{i} \wedge H^{j} \\
& +\frac{1}{2} H^{i} \wedge \nabla_{i}\left(\delta \Gamma_{j k}^{n}\right) \eta_{n} \rho^{j} \wedge \rho^{k}+\delta g_{i \bar{j}} \rho^{i} \wedge * d u^{\bar{j}} \\
& \left.-\delta \Gamma_{i j}^{k} \bar{\eta}^{i} d u^{j} \wedge * A_{k}-\partial_{\bar{\ell}}\left(\delta \Gamma_{i j}^{k}\right) \bar{\eta}^{i} \psi^{\bar{\ell}} \rho^{j} \wedge * A_{k}\right)
\end{aligned}
$$

under $g^{i \bar{j}} \rightarrow g^{i \bar{j}}+\delta g^{i \bar{j}}$.

Similarly to the B model in two dimensions, our theory obviously depends on the variation of complex structures on the target space $X$. Then, moduli space $\mathcal{M}(X)$ of complex structures on $X$ becomes the moduli space of the theory. Thus we may obtain family of smooth invariants of the four manifold parametrized by the moduli space of complex structures of $X$.

\subsection{Localization and Fermionic Zero Modes}

Rescaling $\mathscr{L}$ by $t \mathscr{L}$, the relation (2.25) also implies that the theory is independent of $t$ as long as $\Re t>0$. Thus we may take the semi-classical limit $\Re t \rightarrow \infty$ such that the path integral has dominant contribution from the minima of the bosonic part of the action functional. Such minima are the constant maps $d u^{i}=0$. Thus the path integral is localized to the space of constant maps, which is a copy of the target manifold $X$ itself. Then one may perform perturbative calculation after expanding around constant maps, while taking care of bosonic and fermionic zero-modes. In the one loop computation we will encounter with fermion determinant. We note that the zero forms $\psi^{\bar{i}}$ and self-dual two-forms $g^{\bar{j} i} \chi_{i}$ are sections of $\overline{\mathcal{T}}=T_{X}^{0,1}$, while the one-forms $\rho^{i}$ are sections of $\mathcal{T}=T_{X}^{1,0}$. Consequently the fermion determinant is complex. The situation is similar to the $\mathrm{B}$ model in two dimensions and the condition to have well-defined fermion determinant is that $c_{1}(X)=0, X$ is a Calabi-Yau manifold.

Expanding around the constant map $M \rightarrow X$ the zero modes of the fermions $\psi^{\bar{i}}$ with $U=1$ are the constant modes of $\psi^{\bar{i}}$, i.e., $d \psi^{\bar{i}}$, the zero-modes of $\rho^{i}$ with $U=-1$ are the harmonic one-forms, i.e., $d^{+} \rho^{i}=d * \rho^{i}=0$ and the zero modes of $\chi_{i}$ with $U=1$ are the self-dual harmonic two-forms, i.e., $d^{*+} \chi_{i}=0$. Thus those zero-modes are harmonic representatives of cohomology classes of the following complex

$$
0 \longrightarrow \Omega^{0}(M) \stackrel{d}{\longrightarrow} \Omega^{1}(M) \stackrel{d^{+}}{\longrightarrow} \Omega_{+}^{2}(M) \rightarrow 0,
$$


where $d^{+}=P^{+} \circ d$ and $P^{+}: \Omega^{2}(M) \rightarrow \Omega_{+}^{2}(M)$. Note that the zero-modes of $\eta_{i}$ with $U=3$ and the zero-modes of $\bar{\eta}^{i}$ with $U=-3$ always appear in the pairs. Thus the ghost number anomaly (the net violation of the ghost number in the path integral measure due to the fermionic zero-modes) are

$$
\# U=d\left(1-b_{1}(M)+b_{+}^{2}(M)\right)=\frac{d}{2}(\chi(M)+\sigma(M))
$$

where $b_{1}(M)$ denotes the first Betti number of $M$ and $b_{+}^{2}(M)$ the number of the positive eigen values in the intersection matrix of $H_{2}(M), \chi(M)=2\left(1-b_{1}(M)\right)+b_{2}(M)$ is the Euler number of $M$ and $\sigma(M)=b_{+}^{2}(M)-b_{-}^{2}(M)$ is the signature. It follows that the partition function of the theory is well-defined if and only if the four manifold $M$ has $\chi(M)+\sigma(M)=0$. Otherwise we have to insert suitable combination of observables to have non-vanishing correlation functions.

\subsection{The Observables}

Now we discuss the observables of the theory, which are defined as representatives of $Q$ cohomology classes. The situation will be quite similar to that of topological string B model in the paper [1], and we will follow Witten's discussions there closely.

Consider the following transformation laws from (2.14) and (2.15);

$$
\begin{aligned}
Q u^{i}=0, & Q u^{\bar{i}}=\psi^{\bar{i}}, \\
Q \eta_{i}=0, & Q \psi^{\bar{i}}=0,
\end{aligned}
$$

where the field $\psi^{\bar{i}}$ is a section of $\phi^{*}(\overline{\mathcal{T}})$ of $U=1$ and the field $\eta_{i}$ is a section of $\phi^{*}\left(\mathcal{T}^{*}\right)$ of $U=3$. On the target space $X$ we have the Dolbeault complex $\left(\Omega^{0, \bullet}(X, \wedge \bullet \mathcal{T}), \bar{\partial}\right)$ for the sheaf cohomology group $H_{\bar{\partial}}^{0, \bullet}\left(X, \wedge^{\bullet} \mathcal{T}\right)$. For any $(0, p)$-form $W$ on $X$ with value in $\wedge^{q} \mathcal{T}$, $W \in \Omega^{0, p}\left(X, \wedge^{q} \mathcal{T}\right)$ we associate an operator

$$
\mathcal{O}_{W}^{(0)}=\frac{1}{p ! q !} \psi^{\bar{j}_{1}} \ldots \psi^{\bar{j}_{p}} W_{\bar{j}_{1} \ldots \bar{j}_{p}}^{i_{1} \ldots i_{q}} \eta_{i_{1}} \ldots \eta_{i_{q}}
$$

with the ghost number $U=p+3 q$ Then we find that

$$
Q \mathcal{O}_{W}^{(0)}=\mathcal{O}_{\bar{\partial} W}^{(0)} .
$$

Thus for any representative $W$ of the sheaf cohomology class $[W] \in H_{\bar{\partial}}^{0, p}\left(X, \wedge^{q} \mathcal{T}\right)$ we have an observable $\mathcal{O}_{W}^{(0)}$ of $U=p+3 q$, which is a representative of non-trivial $Q$-cohomology class. Such observables are 0 -forms on $M$ and to be inserted on points in $M$. The analogy with the topological string $B$-model is obvious.

\section{Toward the Topological Family}

In this section we discuss the possibility of constructing more general family of topological Lagrangians. 
The basic idea is very simple; assume that there is a representative of $Q$-cohomology class, which is given by integral of a 4 -form $\mathcal{O}^{(4)}$ on $M$ over $M$, i.e., $\int_{M} \mathcal{O}^{(4)}$, then we may get an one parameter family of topological Lagrangians,

$$
\mathscr{L} \longrightarrow \mathscr{L}+t \int_{M} \mathcal{O}^{(4)}
$$

invariant under $Q$. Obviously we may also consider multiple parameters family by including every $Q$-cohomology class, which representative is the similar integral over $M$. The standard procedure for finding such cohomology classes is using so called the descent equations. To begin with we fix a homogeneous basis $\left\{\left[\gamma_{a}\right]\right\}$ of $H_{\bar{\partial}}^{0, \bullet}\left(X, \wedge^{\bullet} \mathcal{T}\right)$. Let $\left\{\gamma_{a}\right\}$ be a set of representatives and let $\left\{\mathcal{O}_{a}^{(0)}\right\}$ be the associated set of representatives of $Q$-cohomology classes which are 0 -forms on $M$. Then one wants to solve the following sequence of equations called the descent equations;

$$
\begin{aligned}
0 & =Q \mathcal{O}_{a}^{(0)}, \\
d \mathcal{O}_{a}^{(0)} & =Q \mathcal{O}_{a}^{(1)}, \\
d \mathcal{O}_{a}^{(1)} & =Q \mathcal{O}_{a}^{(2)}, \\
d \mathcal{O}_{a}^{(2)} & =Q \mathcal{O}_{a}^{(3)}, \\
d \mathcal{O}_{a}^{(3)} & =Q \mathcal{O}_{a}^{(4)},
\end{aligned}
$$

where $\mathcal{O}_{a}^{(r)}$ is an $r$-form on $M$ with the ghost number $U\left(\mathcal{O}_{a}^{(r)}\right)=U\left(\mathcal{O}_{a}^{(0)}\right)-r$. Let's assume, temporarily, that we have solutions of the above set of equations. Then, the second equation implies that the path integral of observable $\mathcal{O}_{a}^{(0)}$ does not depend on its position at $M$, to where $\mathcal{O}_{a}^{(0)}$ is inserted. In general we pick an $r$-cycle $C_{(r)}$ on $M$ and consider $\int_{C_{(r)}} \mathcal{O}_{a}^{(r)}$. From (3.1), we have

$$
Q \int_{C_{(r)}} \mathcal{O}_{a}^{(r)}=\int_{C_{(r)}} d \mathcal{O}_{a}^{(r-1)}=\int_{\partial C_{(r)}} \mathcal{O}_{a}^{(r-1)}
$$

which relation means that the $Q$ cohomology of $\int_{C_{(r)}} \mathcal{O}_{a}^{(r)}$ depends only on the homology of $C_{(r)}$. In particular for a homology cycle $C_{(r)}, \int_{C_{(r)}} \mathcal{O}_{a}^{(r)}$ gives a non-trivial $Q$-cohomology class. Furthermore one may add $\int_{M} \mathcal{O}_{a}^{(4)}$ to the original Lagrangian $\mathscr{L}$ and get a family of topological Lagrangian. For this we may introduce graded parameters $\left\{t^{\alpha}\right\}$ regarded as the dual basis of $H_{\bar{\partial}}^{0, \bullet}(X, \wedge \bullet \mathcal{T})$ - dual to the homogeneous basis $\left\{\left[\gamma_{\alpha}\right]\right\}$, after shifting ghost number by 4 such that $U\left(t^{a}\right)+U\left(\mathcal{O}_{a}^{(4)}\right)=0$. Then the desired family of Lagrangian $\mathscr{L}_{t}$ is

$$
\mathscr{L}_{t}=\mathscr{L}+t^{a} \int_{M} \mathcal{O}_{a}^{(4)}
$$

of $U=0$. We shall, however, see that we can not solve the descent equations completely.

It is rather straightforward to find $\mathcal{O}_{a}^{(1)}$ solving the second equation in (3.1). We first note that

$$
d \mathcal{O}_{a}^{(0)}=d u^{i} \frac{\delta \mathcal{O}_{a}^{(0)}}{\delta u^{i}}+d \eta_{i} \frac{\delta \mathcal{O}_{a}^{(0)}}{\delta \eta_{i}}+d \psi^{\bar{i}} \frac{\delta \mathcal{O}_{a}^{(0)}}{\delta \psi^{\bar{i}}} .
$$


We also note that the fermionic transformation laws (2.14) and (2.15) imply the following relations

$$
Q \rho^{i}=d u^{i}, \quad Q\left(w_{i}+\Gamma^{k}{ }_{i j} \eta_{k} \rho^{j}\right)=-d \eta_{i}, \quad Q d u^{\bar{i}}=d \psi^{\bar{i}}
$$

as well as

$$
\left[Q, \frac{\delta}{\delta u^{i}}\right]=0, \quad\left[Q, \frac{\delta}{\delta u^{\bar{i}}}\right]=0, \quad\left\{Q, \frac{\delta}{\delta \eta^{i}}\right\}=0, \quad\left\{Q, \frac{\delta}{\delta \psi^{\bar{i}}}\right\}=\frac{\delta}{\delta u^{\bar{i}}}
$$

Now using $Q \mathcal{O}_{a}^{(0)}=0$ and the relations (3.5) and (3.6) we deduce that $\mathcal{O}_{a}^{(1)}$ given by

$$
\mathcal{O}_{a}^{(1)}=\rho^{i} \frac{\delta \mathcal{O}_{a}^{(0)}}{\delta u^{i}}-\left(w_{i}+\Gamma^{k}{ }_{i j} \eta_{k} \rho^{j}\right) \frac{\delta \mathcal{O}_{a}^{(0)}}{\delta \eta_{i}}+d u^{i} \frac{\delta \mathcal{O}_{a}^{(0)}}{\delta \psi^{\bar{i}}},
$$

satisfies $Q \mathcal{O}_{\alpha}^{(1)}=d \mathcal{O}_{\alpha}^{(0)}$. One may proceed to find $\mathcal{O}_{a}^{(2)}$ in the similar fashion. After a tedious but straightforward computations we obtain the following expression

$$
\begin{aligned}
\mathcal{O}_{a}^{(2)}= & -\left(H^{i}+\frac{1}{2} \Gamma^{i}{ }_{j k}\left(\rho^{j} \wedge \rho^{k}\right)^{+}\right) \frac{\delta \mathcal{O}_{a}^{(0)}}{\delta u^{i}} \\
& -\left(\chi_{i}-\Gamma^{k}{ }_{i j}\left(w_{k} \wedge \rho^{j}\right)^{+}+\Gamma^{k}{ }_{i j} \eta_{k} H^{j}-\frac{1}{2} \partial_{i} \Gamma^{\ell}{ }_{j k} \eta_{\ell}\left(\rho^{j} \wedge \rho^{k}\right)^{+}\right) \frac{\delta \mathcal{O}_{a}^{(0)}}{\delta \eta_{i}} \\
& +\frac{1}{2} \rho^{i} \wedge \rho^{j} \frac{\delta^{2} \mathcal{O}_{a}^{(0)}}{\delta u^{j} \delta u^{i}}+\frac{1}{2}\left(w_{i}+\Gamma^{k}{ }_{i m} \eta_{k} \rho^{m}\right) \wedge\left(w_{j}+\Gamma^{\ell}{ }_{j n} \eta_{\ell} \rho^{n}\right) \frac{\delta^{2} \mathcal{O}_{a}^{(0)}}{\delta \eta_{j} \delta \eta_{i}} \\
& +\frac{1}{2} d u^{\bar{i}} \wedge d u^{\bar{j}} \frac{\delta^{2} \mathcal{O}_{a}^{(0)}}{\delta \psi^{\bar{j}} \delta \psi^{\bar{i}}}+\rho^{i} \wedge\left(w_{j}+\Gamma^{\ell}{ }_{j n} \eta_{\ell} \rho^{n}\right) \frac{\delta^{2} \mathcal{O}_{a}^{(0)}}{\delta \eta_{j} \delta u^{i}} \\
& +\rho^{i} \wedge d u^{\bar{j}} \frac{\delta^{2} \mathcal{O}_{a}^{(0)}}{\delta \psi^{\bar{j}} \delta u^{i}}+\rho^{i} \wedge d u^{\bar{j}} \frac{\delta^{2} \mathcal{O}_{a}^{(0)}}{\delta \psi^{\bar{j}} \delta \eta_{i}}
\end{aligned}
$$

satisfies the relation $Q \mathcal{O}_{a}^{(2)}=d \mathcal{O}_{a}^{(1)}$.

It turns out that a solution of the next descent equation $d \mathcal{O}_{a}^{(2)}=Q \mathcal{O}_{a}^{(3)}$ for $\mathcal{O}_{a}^{(3)}$ does not exist in the strict sense. There is, however, a solution $\mathcal{O}_{a}^{(3)}$ of the descent equation modulo the equations of motion;

$$
d \mathcal{O}_{a}^{(2)}=Q \mathcal{O}_{a}^{(3)}+G_{a}
$$

where $G_{a}$ is certain expression that vanishes modulo the $H_{i}$ and $\chi_{i}$ equations of motion. ${ }^{1}$

The similar phenomena had been first observed in the topological string B model [1]. Adopting Witten's recipe there we may proceed further to find $\mathcal{O}_{a}^{(4)}$ satisfying the descent

\footnotetext{
${ }^{1}$ The precise expressions for $\mathcal{O}_{a}^{(3)}$ and $G_{a}$, which are very complicated and unilluminating in the face values, are beyond the purpose of this paper. It is, however, It is not difficult to see what is the origin of the above. Looking back the transformation laws (2.14), we notice that

$$
Q u^{i}=0, \quad Q \rho^{i}=d u^{i}, \quad Q H^{i}=-d^{+} \rho^{i}+\cdots,
$$$$
Q \eta_{i}=0, \quad Q w_{i}=-d \eta_{i}+\cdots, \quad Q \chi_{i}=d^{+} w_{i}+\cdots,
$$

while we do not have 3 -form fields, which the fermionic transformation laws contain $d H^{i}$ and $d \chi_{i}$ terms.
} 
equation modulo equations of motion;

$$
d \bigcup_{a}^{(3)}=Q \mathcal{O}_{a}^{(4)}+\sum_{A} \frac{\delta \mathscr{L}}{\delta \phi_{A}} \cdot \zeta_{a, A}
$$

where $\phi_{A}$ are all the fields of the theory. In the above $\frac{\delta \mathscr{L}}{\delta \phi_{A}}$ are equations of motion, so the 2-nd terms in the right hand side of (3.11) vanishes by the equation. The above relation implies that the first oder deformation toward the topological family

$$
\mathscr{L}^{\prime}=\mathscr{L}+t^{a} \int_{M} \mathcal{O}_{a}^{(4)}
$$

may not be invariant under the original $Q$, but is invariant under the following new $Q^{\prime}$

$$
Q^{\prime} \phi_{A}=Q \phi_{A}+t^{a} \zeta_{a, A}
$$

up to the terms in the second order in $t^{a}$. The new BRST charge $Q^{\prime}$ is also nilpotent only up to the second order. Thus we have to repeat the whole procedure again and again until we fill up all the higher order corrections to both the topological Lagrangian and the BRST charge

$$
\begin{aligned}
\mathscr{L}_{t} & =\mathscr{L}+t^{a} \int_{M} \mathcal{O}_{a}^{(4)}+\sum_{\ell=2}^{\infty} t^{a_{1}} \cdots t^{a_{\ell}} \int_{M} \mathcal{O}_{a_{1} \cdots a_{\ell}}^{(4)}, \\
Q_{t} \phi_{A} & =Q \phi_{A}+t^{a} \zeta_{a, A}+\sum_{\ell=2}^{\infty} t^{a_{1}} \cdots t^{a_{\ell}} \zeta_{a_{1} \cdots a_{\ell}, A},
\end{aligned}
$$

such that $Q_{t} \mathscr{L}_{t}=0$ and $Q_{t}^{2}=0$. Assuming that such all order corrections are possible, the above discussion implies that we have certain extended moduli space $\mathcal{N}$ of the theory such that tangent space to $\mathcal{N}$ at the origin is

$$
\left.T \mathcal{N}\right|_{o} \simeq \bigoplus_{p, q=0}^{d} H_{\bar{\partial}}^{0, p}\left(X, \wedge^{q} \mathcal{T}\right)
$$

-the space of all $Q$ cohomology of zero-dimensional observables. Then the remaining problem is to determine $\mathcal{N}$ and construct the family of topological theories parametrized by $\mathcal{N}$.

We should remark again that the situation is closely related to that of the B model in two-dimensions [1]. We note that the tangent space (3.15) to the moduli space $\mathcal{N}$ is exactly same with the tangent space of the thickened moduli space at a classical point as discussed by Witten for the $\mathrm{B}$ model in two dimensions. We claim that the two moduli spaces are isomorphic to the extended moduli space of complex structures of the Calabi-Yau $X$, defined by Barannikov and Kontsevich [8]. The second named author showed that the extended moduli space of complex structure is the thickennd moduli space parametrizing the family of B-models by actually constructing such a family in Section 4.2 of [9].

The solution of our problem should be obtained by going beyond the on-shell and adopting more powerful viewpoints as was advocated in the papers [9, 10]. The basic strategy is to recast our original Lagrangian $\mathscr{L}$ as a gauge fixed version of certain master 
action functional $S$, adopting the Batalin-Vilkovisky quantization scheme [11, 12]. Then our model is interpreted as a theory of maps

$$
\Phi: T[1] M \longrightarrow \overline{\mathcal{T}}[1] \oplus \mathcal{T}^{*}[3]
$$

where $T[1] M$ is the superspace obtained from the total space of tangent bundle to $M$ after twisting the fiber by $U=1, \overline{\mathcal{T}}[1]$ be the total space of the anti-holomorphic tangent bundle $\overline{\mathcal{T}}=T^{0,1} X$ to the Calabi-Yau space $X$ after twisting the fiber by $U=1$ and $\mathcal{T}^{*}[3]$ be the total space of the holomorphic cotangent bundle $\mathcal{T}^{*}=T^{* 1,0} X$ to $X$ after twisting the fiber by $U=3$. Then one can construct a BV action functional $S$ from a smooth structure on $M$ and the complex structure on $X$, satisfying so called the classical master equation $(S, S)=0$, such that our Lagrangian $\mathscr{L}$ is obtained after gauge fixing $\mathscr{L}=S^{g . f}$. and the BRST charge $Q$ is give by $Q \phi_{A}=\left(S, \phi_{A}\right)^{g . f}$.

The standard description of the extended moduli space $\mathcal{N}$ of complex structures [8] shall be rephrased by extended moduli space associated with the natural differential graded Lie 3 -algebra $(\mathfrak{t}, \bar{\partial},[\bullet, \bullet])$, where $\mathfrak{t}=\bigoplus_{r=0}^{4 n}$ the space of functions on $\overline{\mathcal{T}}[1] \oplus \mathcal{T}^{*}[3]$ - isomorphic to

$$
\mathfrak{t}^{r} \simeq \bigoplus_{r=p+3 q} \Omega^{0, p}\left(X, \wedge^{q} \mathcal{T}\right)
$$

$\bar{\partial}$ is the Dolbeault operator for the given complex structure - regarded as an odd vector field of $U=1$ on $\overline{\mathcal{T}}[1] \oplus \mathcal{T}^{*}[3]$, and the bracket $[\bullet, \bullet]$ is the graded Poisson bracket of $U=-3$ associated with $\mathcal{T}^{*}[3]$ extended naturally to $\mathfrak{t}$. Let $\mathfrak{h}$ be the cohomology of the complex $(\mathfrak{t}, \bar{\partial})$ and let $\left[\gamma_{\alpha}\right]$ be a homogenous basis of $\mathfrak{h}$. Let $\left\{t^{\alpha}\right\}$ be dual to $\left[\gamma_{\alpha}\right]$ after shifting $U$ by 4 in the sense that $U\left(t^{\alpha}\right)=-U\left(\left[\gamma_{\alpha}\right]\right)+4$. Now consider another differential graded Lie 3 -algebra $\left(\mathbb{k}\left[\left[t^{\alpha}\right]\right] \otimes \mathfrak{t}, \mathbf{1} \otimes \partial,[\bullet, \bullet]\right)$. Then a result of $[\mathbb{8}]$ also implies that we have a versal solution $\Gamma \in\left(\mathbb{k}\left[\left[t^{\alpha}\right]\right] \otimes \mathfrak{t}\right)^{4}$ to the Maurer-Cartan equation

$$
\bar{\partial} \Gamma+\frac{1}{2}[\Gamma, \Gamma]=0,
$$

in the form

$$
\Gamma=t^{a} \gamma_{a}+\sum_{\ell=2}^{\infty} \frac{1}{\ell !} t^{a_{1}} \cdots t^{a_{\ell}} \gamma_{a_{1} \cdots a_{\ell}}
$$

where $\left\{\gamma_{a}\right\}$ is a set of representative of $\left\{\left[\gamma_{a}\right]\right\}$ and $\gamma_{a_{1} \cdots a_{n}} \in \mathfrak{t}^{\sum_{k=1}^{\ell} U\left(\gamma_{a_{k}}\right)-4 \ell}$. The MaurerCartan equation (3.16) implies that $\bar{\partial}_{\Gamma}:=\bar{\partial}+[\Gamma$,$] satisfies \bar{\partial}_{\Gamma}^{2}=0$. We claim that the associated BV action functional $S_{\Gamma}$ defined as follows

$$
\begin{aligned}
S_{\Gamma} & :=S+\int_{T[1] M} \Phi^{*}(\Gamma) \\
& =S+t^{\alpha} \int_{T[1] M} \Phi^{*}\left(\gamma_{\alpha}\right)+\sum_{\ell=2}^{\infty} \frac{1}{\ell !} t^{a_{1}} \cdots t^{a_{\ell}} \int_{T[1] M} \Phi^{*}\left(\gamma_{a_{1} \cdots a_{\ell}}\right),
\end{aligned}
$$

where $\int_{T[1] M}$ is the superspace integration over $T[1] M$, also satisfies the classical BV master equation

$$
\left(S_{\Gamma}, S_{\Gamma}\right)=0
$$


Our claim is, then, $S_{\Gamma}$ gives both the desired topological Lagrangian $\mathscr{L}_{t}$ and the BRST charge $Q_{t}$ in the form of (3.14) parametrized by $\mathcal{N}$ after a gauge fixing - i.e., $\mathscr{L}_{t}=\left(S_{\Gamma}\right)^{g . f}$. such that $S^{g . f .}=\mathscr{L}$ and $\left(\int_{T[1] M} \Phi^{*}\left(\gamma_{a_{1} \cdots a_{\ell}}\right)\right)^{g . f .}=\int_{M} \mathcal{O}_{a_{1} \cdots a_{\ell}}^{(4)}$ for all $\ell=1,2,3, \ldots$ and $Q_{t} \phi_{A}=\left(S_{\Gamma}, \phi_{A}\right)^{g . f .}$. Then the desired relations that $Q_{t}^{2}=0$ and $Q_{t} \mathscr{L}_{t}=0$ are satisfied as the results of the BV master equation (3.19). Furthermore this approach also leads to stream lined Hamiltonian formalism of the family of theory as was sketched in Sect. 4.1 of [10] for general case. The details of these discussions shall appear elsewhere [13].

\section{Acknowledgments}

We would like to thank Hoil Kim for useful discussions. We also like to thank the referee whose comments helped us improve the presentation of this paper.

\section{References}

[1] E. Witten, Mirror manifolds and topological field theory, hep-th/9112056.

[2] E. Witten, Topological quantum field theory, Commun. Math. Phys. 117 (1988) 353

[3] S.K. Donaldson, Polynomial invariants for smooth 4-manifolds, Topology 29 (1990) 257.

[4] H.-K. Jun and J.-S. Park, Holomorphic Symplectic Geometry and Smooth 4-Manifolds, in preparation.

[5] L. Rozansky and E. Witten, Hyper-Kaehler geometry and invariants of three-manifolds, Selecta Math. 3 (1997) 401. hep-th/9612216.

[6] M. Kontsevich, Rozansky-Witten invariants via formal geometry, Compositio Math. 115 (1999) 115.

[7] M. Kapranov, Rozansky-Witten invariants via Atiyah classes. Compositio Math. 1999 (71).

[8] S. Barannikov and M. Kontsevich, Frobenius manifolds and formality of Lie algebras of polyvector fields. Internat. Math. Res. Notices 4 (1998) 201. alg-geom/9710032.

[9] J.-S. Park, Topological open p-branes, Proceedings of the KIAS conference Symplectic Geometry and Mirror Symmetry, pp. 311-384, ed. by Fukaya, Oh, Ono, Tian. World Scientific 2001. hep-th/0012141.

[10] J.-S. Park, Pursuing the quantum world: Flat family of QFTs and quantization of $d$-algebras, hep-th/0308130.

[11] I.A. Batalin and G.A. Vilkovisky, Gauge algebra and quantization, Phys. Lett. B 102 (1981) 27 .

[12] M. Alexandrov, M. Kontsevich, A. Schwartz and O. Zaboronsky, The geometry of the master equation and topological quantum field theory, Int. J. Mod. Phys. A 12 (1997) 1405, hep-th/9502010.

[13] H.-K. Jun and J.-S. Park, 4-algebras, smooth 4-manifolds and TFTs in 4-dimensions, in preparation. 